\title{
THREE-DIMENSIONAL VALIDATION EXERCISE FOR FIESTA CODE: EVOLUTION OF SHOCK-DRIVEN INSTABILITIES
}

\author{
BRIAN ROMERO ${ }^{1,2}$, PETER VOROBIEFF $^{1}$, SVETLANA V. POROSEVA $^{1}$ \& JON M. REISNER ${ }^{2}$ \\ ${ }^{1}$ University of New Mexico, USA \\ ${ }^{2}$ Los Alamos National Laboratory, USA
}

\begin{abstract}
In this paper, we present simulation results for the three-dimensional, shock-driven Kelvin-Helmholtz instability. Simulations are performed with a Mach 2.0 shock propagating through a finite-diameter cylindrical column of dense gas inclined at an angle $\theta$ with respect to the shock plane. After passage of the shock, the gas curtain has accelerated along its axis and a Kelvin-Helmholtz instability forms on the column surface. This is the first known numerical reproduction of this phenomena in three dimensions, which has previously been observed in experiments with an inclined cylindrical gas column. The effects of changes to initial column angle $\left(\theta=0^{\circ}, 10^{\circ}, 20^{\circ}, 30^{\circ}\right)$ are explored in detail to complement experimental data. The effects of shock reflection near the base of the column are also examined to identify a possible flow perturbation near the foot which was seen in our previous two-dimensional numerical studies of shock-accelerated inclined gas curtains. The overall flow morphology compares well with experimental data in a cross-sectional plane through the column midpoint and a vertical plane through the column axis. Simulations were performed with FIESTA, an exascale ready, GPU accelerated compressible flow solver developed at the University of New Mexico.

Keywords: multiphase flow, mixing, modeling, compressible flow, validation.
\end{abstract}

\section{INTRODUCTION}

In many problems of fundamental or practical interest, transition to turbulence is driven by a shock propagating through a non-uniform medium, such as gases of different densities, possibly with non-gaseous inclusions in the form of particles or liquid drops. In astrophysics, shocks from supernova explosions and asymptotic giant branch stars propagate through gas and cosmic dust [1]-[3], leading to formation of structures on the scale of light years [4], [5]. In determining the origin of cosmic dust, a factor of great interest is its evaporation in supernovae [6]. In geophysics, shock waves and impulsive acceleration events that strongly affect Earth's magnetosphere occur as the result of interaction between the solar wind and Earth's magnetopause [7]. Engineering applications include fuel mixing in scramjets [8], explosion physics [9], [10], and inertial confinement fusion [11]. The mixing of liquid fuel droplets with air by oblique shock waves creates issues for scramjet engines [12]. An understanding of detonation waves with a multiphase air-fuel droplet mixture is essential for designing more practical liquid fueled pulse detonation and rotating detonation engines [13].

There are three well-known interfacial instabilities: Kelvin-Helmholtz (KHI), driven by shear, Rayleigh-Taylor (RTI), driven by sustained acceleration in the direction of decreasing density across the interface, and Richtmyer-Meshkov (RMI), driven by impulsive acceleration across the interface. It is possible to set up a problem where one of these instabilities would clearly dominate, but in many situations of practical interest, all three may play some role. The situation is further complicated if the medium of interest is multiphase. In the simpler case, a multiphase medium would be comprised of a single phase occupying a dominant volume fraction, with small inclusions of the second phase (droplets or particles in gas, bubbles in fluid), as even a small volume fraction can have a large fraction of the total mass. Droplets or particles carried by the gas flow strongly change the relevant physics, e.g., 
by changing the compressibility of the medium (fluid with bubbles) or its average density. Moreover, for the case of shock-accelerated gas carrying particles or droplets, it cannot be safely assumed that the inclusions remain embedded in the post-shock flow. As the particles increase in size they become slow to react and the length and time scales of the effects of their interaction with the surrounding medium must be carefully considered. For droplets, break-up or coagulation may be precipitated by the shock passage. The addition of phase change and reactions also influences the evolution of the flow greatly and brings additional complexity to the problem.

Numerical modeling of shock-driven compressible flow, especially when the medium is multispecies and/or multiphase, remains a challenging problem which cannot be solved with a brute-force approach despite the recent advances in computational power. The range of scales that need to be resolved would be prohibitively computationally expensive - from molecular mean free path (representative value $60 \mathrm{~nm}$ for nitrogen at atmospheric pressure) to maximum system scale (which could be light-years for astrophysical problems). Within this range spanning multiple orders of magnitude, different physics would manifest at different scales: the shock front thickness is on the same order as the mean free paths, the Kolmogorov microscales at and below which viscous dissipation becomes dominant are typically in the micron range, the scales associated with particle/droplet interaction are commensurate with the particle/droplet sizes, and so on. Accordingly, in most cases the computational scheme is organized in some way that does not directly resolve the smallest scales - commonly by timeaveraging small-scale fluctuations, leading to Reynolds-averaged Navier-Stokes equations (RANS), or by low-pass filtering of the Navier-Stokes equations, resulting in LES (largeeddy simulation) computational schemes. In both cases, additional conjectures are required to account for extra variables introduced due to averaging (RANS, closure models) or to model subgrid scales (LES).

Another approach is modeling full Navier-Stokes (or Euler, if viscosity is to be disregarded) equations without resolving all the scales, but with no subgrid model [14]. This approach is known as implicit LES (ILES). Here we present a validation exercise for a newly developed, open-source code modeling Euler equations and resolving the flow structure on scales about an order of magnitude larger that the Kolmogorov microscale. The goal of the study is twofold: confirm that the code faithfully reproduces experimental results and gain insights into the flow by analyzing numerically modeled features that cannot be tracked with available experimental diagnostics.

\section{EXPERIMENTAL ARRANGEMENT AND VALIDATION DATA}

The experiments were conducted at the University of New Mexico tiltable shock tube [15][17]. Prior to release of the shock, the optically transparent test section of the shock tube is maintained at atmospheric pressure. A cylindrical jet of heavy gas (a mixture of sulfur hexafluoride $\mathrm{SF}_{6}$ and acetone with traces of air) is injected downward through the top wall of the test section and exits through a hole in the bottom wall. This jet is surrounded with a co-flowing cylindrical jet of air, so that the flow is laminar and any mixing between the air (density $\rho_{1}$ ) and heavy gas (density $\rho_{2}$ ) is diffusive. The entire shock tube can be tilted to an angle $\theta$ with respect to the horizontal. With a column injection system chosen for a specific $\theta$, the jet with nominal diameter $D_{I C}$ flows as shown in Fig. 1.

Once these initial conditions with a cylindrical diffuse density interface are established, the shock tube produces a planar shock wave at a prescribed Mach number $M$. The shock front propagates from the driver section (not shown in Fig. 1) toward the test section. Behind the shock front the air is compressed and moving with piston velocity $\Delta V$. 


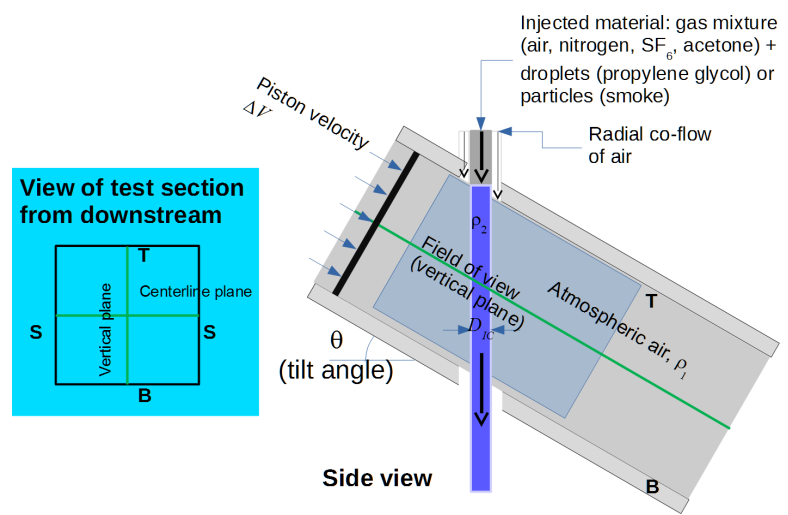

Figure 1: Schematic of the experimental arrangement for studies of shock interaction with an inclined cylindrical density interface (same setup as [16], [17]). Walls of the test section of the shock tube (and the corresponding computational domain) are labeled as $\mathrm{T}$ - top, $\mathrm{B}$ - bottom, and $\mathrm{S}$ - side.

During the experiments, pressure traces are collected at multiple downstream locations to monitor the shock propagation and trigger the imaging system, which can capture images in two planes. The first visualization plane (vertical) is parallel to the shock direction and equidistant from the vertical walls of the test section. The second plane (centerline) is similarly parallel to the top and bottom walls and tilted at the same angle $\theta$ as the shock tube. Each plane can be illuminated with precisely timed, short $(\sim 5 \mathrm{~ns})$ pulses of a frequency-quadrupled (wavelength $266 \mathrm{~nm}$ ) Nd:YAG laser. The laser beam passes through a combination of a cylindrical and a spherical lens, forming a laser sheet that selectively illuminates a narrow (submillimeter) planar cross-section of the flow. The acetone in the injected heavy gas mixture fluoresces in the visible range at $480 \mathrm{~nm}$ when lit with the 266nm laser pulse.

The governing parameters of the flow are $D_{I C}, \theta, M$ and the Atwood number

$$
A=\frac{\rho_{2}-\rho_{1}}{\rho_{2}+\rho_{1}}
$$

Fig. 2 shows an example of an image sequence in two planes. Dimensionless time $\tau$ is defined there in accordance with Richtmyer's linear theory as

$$
\tau=k A \Delta V t
$$

where $t$ is dimensional time and $k=2 \pi / D_{I C}$ is the dominant wavenumber of the initial density perturbation. In terms of $\tau$, the initial RMI growth rates should remain the same for the same initial condition geometry, not changing with $A$ or $M$ (although this assertion is only valid for very early - linear - perturbation growth). The volume in the flow where the perturbed heavy gas mixes with the surrounding lighter gas is commonly referred to as the mixing zone, and its streamwise and spanwise extent can serve as quantitative code-validation benchmarks.

In the centerline plane, the dominant flow feature is a pair of counter-rotating vortices that roll up due to RMI. In the vertical plane, however, small-scale vortices form on the leading and trailing edges of the heavy-gas cylinder [16]. Based on the observed morphology, 

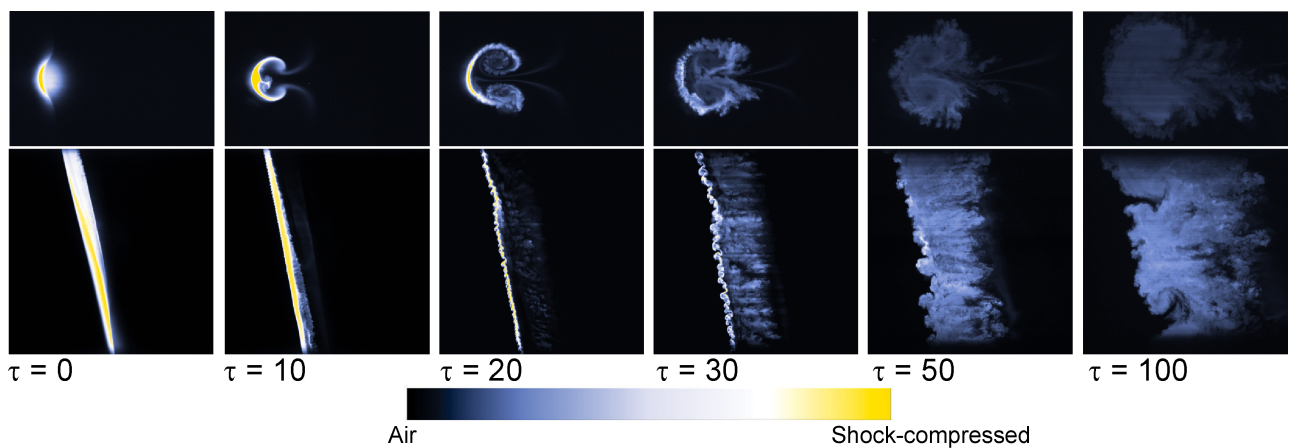

Air $\tau=50$ $\tau=100$

Figure 2: Representative experimental image sequence showing shock acceleration of a gas cylinder at a tilt angle $\theta=20^{\circ}, M=2, A=0.6$ [17]. $\tau$ is dimensionless time (refer to text). The top image for each $\tau$ shows the centerline plane, the bottom image - the vertical plane. Images are shown in false color to emphasize feature formation. Fluorescence intensity (color bar) increases monotonically with acetone (heavy gas tracer) concentration. Streamwise extent of the images is $44 \mathrm{~mm}$, the field of view is following the mixing zone.

it was suggested that the vortex formation mechanism responsible for these features in the vertical plane is KHI due to shock-driven vorticity deposition on the inclined density interfaces [16], [18], [19]. As the flow evolves, secondary instabilities begin to emerge in both planes, leading to enhanced mixing in increasingly disordered flow that at late time manifests statistics consistent with turbulence [17].

The experimental results described above can be used to develop a well-characterized set of quantitative benchmarks for validation of numerical codes modeling shock-driven mixing and /or multphase flow. We begin with a validation exercise for the case of a single-phase density interface, as described in the next section.

\section{NUMERICAL SETUP}

The computational framework for simulations is FIESTA (Fast Interface Evolution, Shocks and Transition in the Atmosphere). It is a new open-source computational fluid dynamics code developed at the University of New Mexico to study instabilities and transition to turbulence. FIESTA is capable of carrying out simulations at scales ranging from those characteristic for a laboratory shock tube to large atmospheric scales.

The code is written in the modern $\mathrm{C}++$ language and takes advantage of object-oriented techniques to improve the code modularity. FIESTA uses the Kokkos C++ Performance Portability Ecosystem [20] to target both traditional CPU architectures and General Purpose Graphics Processing Units (GPGPUs) while avoiding the code complexity and duplication commonly encountered when supporting different architectures. Output files are formatted using the HDF5 file format [21]. Input files are written in the Lua language [22] allowing for easy parameterization of a problem. Multi-GPGPU support is provided by the Message Passing Interface (MPI).

The modular code design allows to include different physics and models as needed. Existing modules can solve two-dimensional (2D) and three-dimensional (3D) two-species Euler and NS equations on generalized curvilinear or uniform Cartesian structured grids. The Euler equations are solved with a simplified 5th-order weighted essentially non-oscillatory 
(WENO5) finite difference scheme [23], [24]. The time scheme used is a low storage, secondorder, explicit Runge-Kutta integrator. At each time level, advection terms are approximated using the WENO5 scheme. The pressure gradient term is approximated using a fourth-order central difference scheme [23], [24].

To obtain the results presented here, we used fully three-dimensional, two-species, inviscid Euler equations in their conservative form. These equations consist of the continuity equations for each gas species, equations for each momentum component, and the conservation equation for specific total energy [25]. The size of the computational domain was $40 \times 10 \times 5 \mathrm{~cm}$ in the $x$ (streamwise), $y$ (vertical) and $z$ (normal to the $x-y$ plane) directions respectively. The equations were solved on a uniform rectangular grid: $d x=d y=d z=67 \mu \mathrm{m}$, with reflective conditions on the top and bottom of the domain, and outflow conditions upstream, downstream and on each side in the $z$-direction. Unlike experiment, where the initial conditions are formed by gravity-driven flow, gravity is not considered, instead the heavy-gas column is tilted to an angle $\theta$ in the computational domain. The column is modeled as initially diffusive, with gas concentration profiles matching experimental measurements [16]. Note that in experiments, the gravity-driven jet of heavy gas has a velocity on the order of centimeters per second prior to shock acceleration - this is disregarded in the modeling, as the representative piston velocity of the shock-accelerated flow at $M=2$ is $434 \mathrm{~m} / \mathrm{s}$.

Dalton's law is assumed to govern the pressure of gas mixtures, and gases are modeled with ideal-gas equation of state, with gas constants $\gamma=1.402$ for air and $\gamma=1.095$ for injected heavy gas (gas constant for $\mathrm{SF}_{6}$ ). The pressure and temperature in the gases prior to shock arrival are set at $300 \mathrm{~K}$ and 1 bar.

\section{RESULTS AND DISCUSSION}

The work presented here is a step forward from the implementation of the 2D module of FIESTA [25], which was used to simulate shock interaction with an inclined gas curtain (not cylinder) and to confirm the two-dimensional inviscid model of shock-driven KHI originally based on experiment [16]. Fig. 3 shows a time sequence of density maps in two planes obtained as a result of the three-dimensional FIESTA run simulating the experiment whose initial conditions are schematically shown in Fig. 1 and results - in Fig. 2. There is good agreement between simulation and experiment - both qualitative and quantitative. The flow features observed in experiment are faithfully reproduced, including the counter-rotating vortices in the centerline plane, the Kelvin-Helmholtz vortices in the vertical plane, and the emergence of secondary instabilities.

Quantitative comparison of experiments and numerics is still ongoing, but preliminary measurements show good agreement in terms of integral flow features such as mixing zone width (extent of the perturbed column in the streamwise direction) and the dominant wavelength of the KHI in the vertical plane [25].

Similar observations can be made with regard to the $\theta=30^{\circ}$ simulation, Fig. 4 . However, the computational exercise we present here goes beyond experimental validation of the $3 \mathrm{D}$ FIESTA module. The diagnostics we use in experiment (local pressure measurements at four points and planar laser-induced Fluorescence - PLIF - imaging in two planes) cannot reveal some important flow features, for example, the 3D geometry of the shock reflected from the inclined gas column ( $\tau=20$ in Fig. 3 and $\tau=14$ in Fig. 4). This pattern is remarkably similar to shock reflection off an elongated solid body, which could offer some hints about the influence of the Mach number on the nonlinear instability growth: it was shown that growth plots for different initial conditions and Mach numbers can be collapsed to a single curve in dimensionless coordinates with time $\tau$ and the instability amplitude nondimensionalized with 


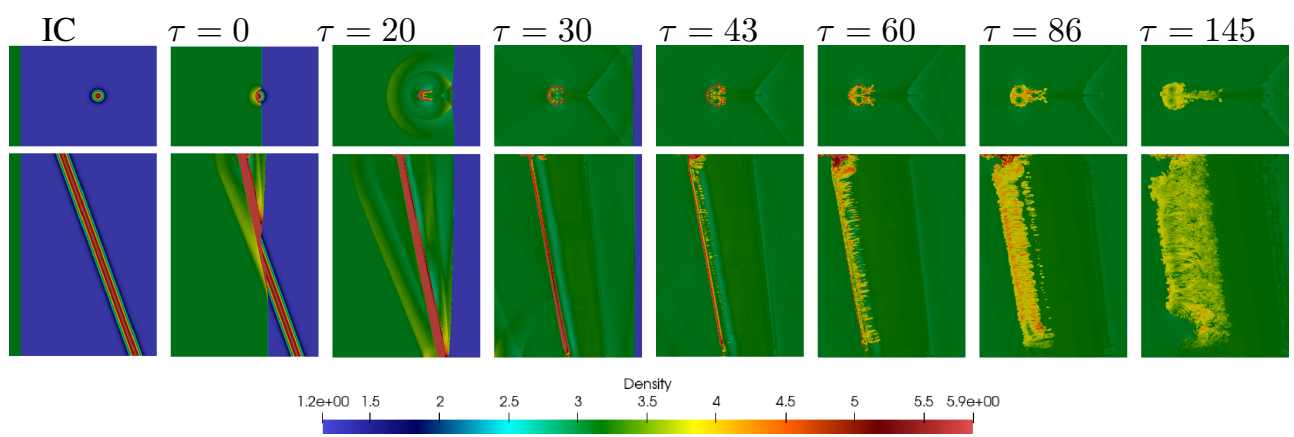

Figure 3: Density maps in the centerline (top row) and vertical (bottom row) planes obtained with FIESTA, $M=2, A=0.6, \theta=30^{\circ}$. Density units (color bar) are $\mathrm{kg} / \mathrm{m}^{3}$. Only a part of the streamwise extent of the computational domain with features of interest is shown, similar to Fig. 2. First image (label "IC") shows the initial conditions. $\tau=0$ corresponds to the time when the shock arrives at the column in the centerline plane.

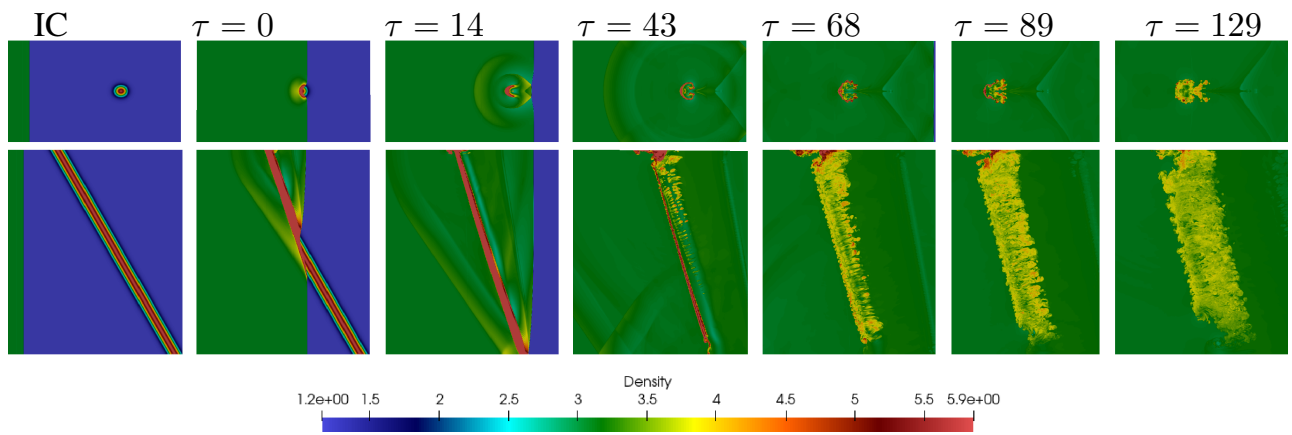

Figure 4: Density maps in the centerline (top row) and vertical (bottom row) planes obtained with FIESTA, $M=2, A=0.6, \theta=30^{\circ}$. Density units (color bar) are $\mathrm{kg} / \mathrm{m}^{3}$. Only a part of the streamwise extent of the computational domain with features of interest is shown. [16], [18], contain corresponding experimental images and data for validation.

a scaling $w_{0} M^{\kappa}$, where $w_{0}$ is the minimal perturbation amplitude after shock compression and phase inversion, and $\kappa \sim 0.5$ [16], [26]. A similar $M^{0.5}$ power-law scaling is known from ballistics [27], [28], so it was conjectured that similar wake-like features in the flows are responsible for the scalings. The simulation results shown here confirm the presence of such features.

While focus on the mixing zone features (Figs 2-4) is important for experimental validation of the code, it is also instructive to examine the entirety of the computational domain. Fig. 5 shows density maps revealing several important features beyond the mixing zone. Reflected waves from shock-column interaction propagate upstream. In 2D simulations of a gas curtain [25], surface-shock interaction near the foot of the inclined curtain was shown to produce a length scale that is amplified by shock-driven KHI. A similar phenomenon is now apparent in the 3D simulation. Downstream, a spike of dense material ejected from the cylinder moves with a high subsonic velocity $(\sim 0.3 M$ in terms of the local Mach 

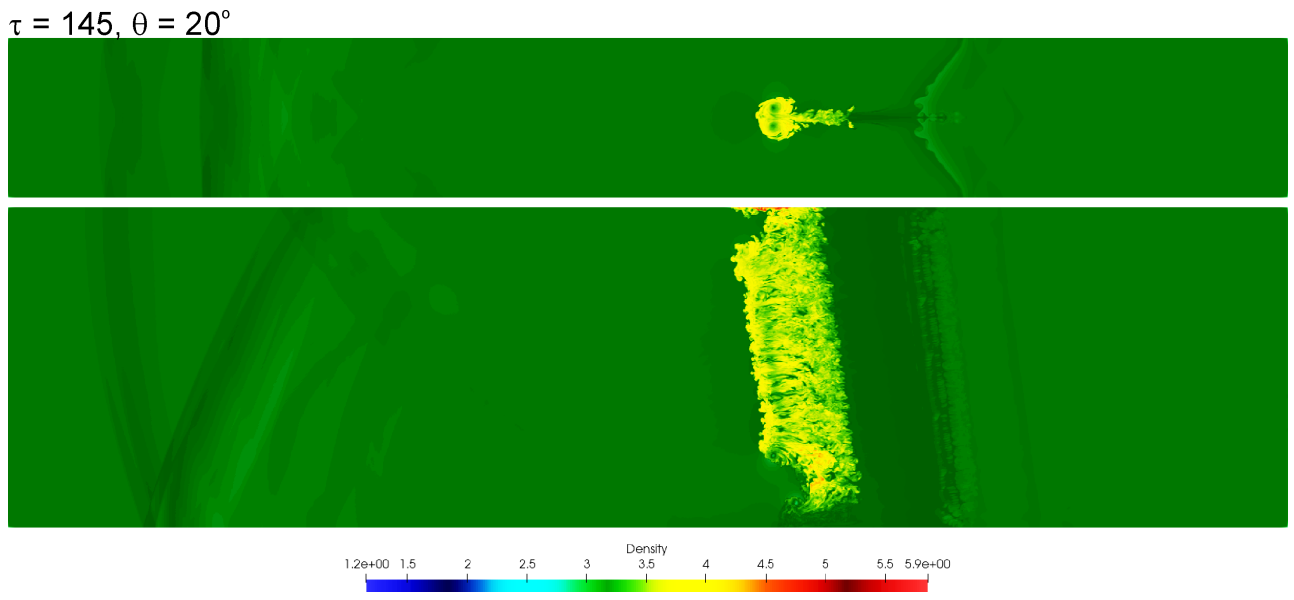

Figure 5: Centerline and vertical plane density maps spanning the entire computational domain at late times. Density units (color bar) are $\mathrm{kg} / \mathrm{m}^{3}$, palette is different from the one used in Figs 3 and 4 to emphasize features outside the mixing zone, including the material ejected from the column in the downstream direction due to shock focusing and pressure wave reflection propagating upstream.

number [29]). Vortex structures at the end of the spike have been observed in experiment ("lion's tail" [29]), however, the adjacent wakelike structure best visible in the centerline plane of Fig. 5 could not be visualized because there was no fluorescent tracer to track it.

\section{CONCLUSIONS AND FUTURE WORK}

Our numerical studies of three-dimensional interaction between a planar shock front and an inclined, initially diffuse column of heavy gas help validate the 3D Euler equations module of the new open-source FIESTA code. Additionally, the modeling reveals important flow features that would be difficult to resolve experimentally with fluorescence-based flow visualization techniques.

Follow-up work with FIESTA will include grid convergence studies, an implementation of a compressible multiphase flow module, and incorporation of different equations of state for single gases and mixtures to account for real-gas effects.

FIESTA validation exercises will be extended beyond integral quantitative benchmarks, such as streamwise mixing zone extent, to robust statistical benchmarks based on velocity and scalar structure function scalings [17], [30], [31], fractal characteristics of the mixing interfaces [32], and species concentration statistics [16].

\section{ACKNOWLEDGEMENTS}

Peter Vorobieff is a Halliburton Professor. The Halliburton Professorship was established in 1982 by the Halliburton Foundation, and the endowment was subsequently expanded by the addition of funds from the State of New Mexico. This research is partially supported by the Defense Threat Reduction Agency (DTRA) grant HDTRA-18-1-0022. Mr. Romero acknowledges support by the New Mexico Consortium. We also wish to thank the UNM Center for Advanced Research Computing, supported in part by the US National Science Foundation, for providing some of the high performance computing resources used in this work. 


\section{REFERENCES}

[1] Mendis, D.A. \& Rosenberg, M., Cosmic dusty plasma. Annual Review of Astronomy and Astrophysics, 32(1), pp. 419-463, 1994.

[2] Bocchio, M., Jones, A.P. \& Slavin, J.D., A re-evaluation of dust processing in supernova shock waves. Astronomy \& Astrophysics, 570, p. A32, 2014.

[3] Woitke, P., 2D models for dust-driven AGB star winds. Astronomy \& Astrophysics, 452(2), pp. 537-549, 2006.

[4] Chevalier, R.A., Blondin, J.M. \& Emmering, R.T., Hydrodynamic instabilities in supernova remnants - Self-similar driven waves. The Astrophysical Journal, 392, pp. 118-130, 1992.

[5] Kane, J., Drake, R. \& Remington, B., An evaluation of the Richtmyer-Meshkov instability in supernova remnant formation. The Astrophysical Journal, 511(1), p. 335, 1999.

[6] Bianchi, S. \& Schneider, R., Dust formation and survival in supernova ejecta. Monthly Notices of the Royal Astronomical Society, 378(3), pp. 973-982, 2007.

[7] Wu, C., Shock wave interaction with the magnetopause. Journal of Geophysical Research: Space Physics, 105(A4), pp. 7533-7543, 2000.

[8] Yang, J., Kubota, T. \& Zukoski, E.E., Applications of shock-induced mixing to supersonic combustion. AIAA Journal, 31(5), pp. 854-862, 1993.

[9] DOE ASCI teraflops computer fully running for first time. Sandia National Laboratories News Release, 1997. http://www.sandia.gov/media/online.htm.

[10] Benjamin, R.F., An experimenter's perspective on validating codes and models with experiments having shock-accelerated fluid interfaces. Computing in Science \& Engineering, 6(5), pp. 40-49, 2004.

[11] Goncharov, V., Theory of the ablative Richtmyer-Meshkov instability. Physical Review Letters, 82(10), p. 2091, 1999.

[12] Pandey, K. \& Sivasakthivel, T., Recent advances in scramjet fuel injection - A review. International Journal of Chemical Engineering and Applications, 1(4), p. 294, 2010.

[13] Huang, Y., Tang, H., Li, J. \& Zhang, C., Studies of DDT enhancement approaches for kerosene-fueled small-scale pulse detonation engines applications. Shock Waves, 22(6), pp. 615-625, 2012.

[14] Margolin, L.G., Rider, W.J. \& Grinstein, F.F., Modeling turbulent flow with implicit LES. Journal of Turbulence, (7), p. N15, 2006.

[15] Anderson, M., Vorobieff, P., Truman, C., Corbin, C., Kuehner, G., Wayne, P., Conroy, J., White, R. \& Kumar, S., An experimental and numerical study of shock interaction with a gas column seeded with droplets. Shock Waves, 25(2), pp. 107-125, 2015.

[16] Olmstead, D., Wayne, P., Yoo, J.H., Kumar, S., Truman, C.R. \& Vorobieff, P., Experimental study of shock-accelerated inclined heavy gas cylinder. Experiments in Fluids, 58(6), p. 71, 2017.

[17] Olmstead, D., Wayne, P., Simons, D., Monje, I.T., Yoo, J.H., Kumar, S., Truman, C.R. $\&$ Vorobieff, P., Shock-driven transition to turbulence: Emergence of power-law scaling. Physical Review Fluids, 2(5), p. 052601, 2017.

[18] Wayne, P., Olmstead, D., Vorobieff, P., Truman, C. \& Kumar, S., Oblique shock interaction with a cylindrical density interface. WIT Transactions on Engineering Sciences, vol. 89, WIT Press: Southampton and Boston, pp. 161-169, 2015.

[19] Romero, B.E., Poroseva, S., Vorobieff, P. \& Reisner, J., Shock driven Kelvin-Helmholtz instability. AIAA Scitech 2021 Forum, p. 0051, 2021. 
[20] Edwards, H.C., Trott, C.R. \& Sunderland, D., Kokkos: Enabling manycore performance portability through polymorphic memory access patterns. Journal of Parallel and Distributed Computing, 74(12), pp. 3202-3216, 2014.

[21] Koranne, S., Hierarchical data format 5: HDF5. Handbook of Open Source Tools, Springer, pp. 191-200, 2011.

[22] Ierusalimschy, R., Programming in Lua, Roberto Ierusalimschy, 2006.

[23] Ramani, R., Reisner, J. \& Shkoller, S., A space-time smooth artificial viscosity method with wavelet noise indicator and shock collision scheme, Part 1: The 1-D case. Journal of Computational Physics, 387, pp. 81-116, 2019.

[24] Ramani, R., Reisner, J. \& Shkoller, S., A space-time smooth artificial viscosity method with wavelet noise indicator and shock collision scheme, Part 2: The 2-D case. Journal of Computational Physics, 387, pp. 45-80, 2019.

[25] Romero, B., Poroseva, S., Vorobieff, P. \& Reisner, J., Simulations of the shock driven Kelvin-Helmholtz instability in inclined gas curtains. Physics of Fluids, 2021. In press.

[26] Orlicz, G., Balasubramanian, S. \& Prestridge, K., Incident shock mach number effects on Richtmyer-Meshkov mixing in a heavy gas layer. Physics of Fluids, 25(11), p. 114101, 2013.

[27] McCoy, R., Modern Exterior Ballistics: The Launch and Flight Dynamics of Symmetric Projectiles, Schiffer Pub., 1999.

[28] Roetzel, W., Analytical calculation of trajectories using a power law for the drag coefficient variation with Mach number. WIT Transactions on Modelling and Simulation, vol. 40, WIT Press: Southampton and Boston, 2005.

[29] Bernard, T., Randall Truman, C., Vorobieff, P., Corbin, C., Wayne, P.J., Kuehner, G., Anderson, M. \& Kumar, S., Observation of the development of secondary features in a Richtmyer-Meshkov instability driven flow. Journal of Fluids Engineering, 137(1), 2015.

[30] Vorobieff, P., Rightley, P.M. \& Benjamin, R.F., Power-law spectra of incipient gascurtain turbulence. Physical Review Letters, 81(11), p. 2240, 1998.

[31] Vorobieff, P., Mohamed, N.G., Tomkins, C., Goodenough, C., Marr-Lyon, M. \& Benjamin, R., Scaling evolution in shock-induced transition to turbulence. Physical Review E, 68(6), p. 065301, 2003.

[32] Vorobieff, P., Rightley, P.M. \& Benjamin, R.F., Shock-driven gas curtain: Fractal dimension evolution in transition to turbulence. Physica D: Nonlinear Phenomena, 133(1-4), pp. 469-476, 1999. 